\title{
Performance Analysis and Simulation of Permanent Magnet Motor Based on Numerical Calculation of Electromagnetic Field
}

\author{
Aiping Deng \\ Pingxiang University, Pingxiang, Jiangxi, China, 337055 \\ 596849661@qq.com
}

Keywords: Permanent Magnet Motor; Performance Analysis; Electromagnetic Field; Numerical Calculation

\begin{abstract}
The permanent magnet motor has the advantages of high power density, safe operation and simple maintenance, and so on. Based on the analysis of the three-dimensional magnetic field distribution characteristics of permanent magnet synchronous motor (PMSM), permanent magnet DC motor is used as an example, The dynamic finite element analysis model of permanent magnet DC motor is characterized by the new interpolation motion boundary method to solve the rotor motion. The commutation law of the winding is calculated according to the actual brush width and winding distribution. Through the finite element analysis model, the current-torque characteristics, taking into account the cogging effect and commutation caused by the torque ripple, in-depth study of permanent magnet DC motor performance is important. The analysis method used in this work can be used to analyze the whole winding permanent magnet motor, can also analyze the ordinary DC motor, the simulation results prove the accuracy of the algorithm, the electromagnetic field numerical calculation method for permanent magnet motor design and performance analysis is important Guiding significance.
\end{abstract}

\section{Introduction}

Permanent magnet DC motor has both good excitation and DC motor speed characteristics and mechanical characteristics, but also eliminates the need for field winding and has a simple structure, small size, with less copper, high efficiency. The total cost of using ferrite permanent magnets is generally lower than that of an electromagnetism motor. From the home appliances, portable electronic devices, power tools to require a good dynamic performance of the precision speed and position of the transmission system are widely used permanent magnet DC motor. Permanent magnet DC motor has a broad application of development space. In Ref.1, the principle, design and stabilization system of permanent magnet DC motor are introduced in detail. In this work, a series of studies on the performance analysis of permanent magnet DC motor are carried out by field-circuit method. The effective length of the armature, a series of data for magnetic circuit calculation, the accuracy of magnetic circuit analysis is improved.

There are two key problems in the dynamic finite element model of permanent magnet motor. First, the rotor motion problem is simple and efficient, and the second is the accurate calculation of the winding current distribution law. The dynamic finite element model is actually a time-step finite element method. If the rotor motion problem is less efficient, it will bring a lot of computational load, or only a few rotors the performance of the motor can not be accurately calculated when calculating. In the process of rotor movement, the winding current changes due to commutation, so to calculate the rotation of the armature on the winding current changes over time, which is not involved in the static analysis model ${ }^{[2-3]}$.

Aiming at the above research situation, this work establishes a precise dynamic finite element analysis model of permanent magnet DC motor. A new type of interpolation motion boundary method is used to deal with the relative motion of the rotor. For the wide range of three-slot motors, the difference of the armature reaction between the forward and reverse rotation of the star-connected three-slot motor is pointed out and the reason is revealed by comparing the armature reaction law of the winding connection and the winding triangular connection. The causes of the torque ripple caused 
by the permanent magnet motor are analyzed, and the measures to reduce the torque ripple are discussed. A simple and efficient calculation method for cogging torque is given to the chute and the oblique pole, and the measures to establish the sine wave air gap magnetic field to weaken the torque ripple are discussed ${ }^{[4-5]}$.

\section{Mathematical model of magnetic field of permanent magnet motor}

Permanent magnet permanent treatment method is the permanent magnet magnetization characteristics directly reflected in the differential equation. The permanent magnet works on the reply line and the return line is a straight line. For rare earth magnets, the return lines and demagnetization curves coincide. The relationship between $\mathrm{B}$ and $\mathrm{H}$ of the permanent magnet operating point is ${ }^{[6-7]}$ :

$$
H=H_{c}-\frac{1}{\mu} B
$$

Where $\mu=\mu_{0} \mu_{c}$

Using the vector magnetic bit A as the solution of the bit function, according to the Maxwell equation, the electromagnetic field boundary value of the permanent magnet motor can be expressed as:

$$
\left\{\begin{array}{l}
\nabla \times\left(\frac{1}{\mu} \nabla \times A\right)=J_{c} \\
\nabla \times\left(H_{c}-\frac{1}{\mu} \nabla \times A\right)=0
\end{array}\right.
$$

This boundary value problem can be equivalent to:

$$
W(A)=\int_{V_{1}} \frac{1}{2 \mu}(\nabla \times A)^{2} d \tau+\int_{V_{2}} \frac{1}{2 \mu}\left(\mu H_{c}-\nabla \times A\right)^{2} d \tau-\int_{V_{1}} J_{L} \cdot A d \tau=\min
$$

In a two-dimensional field, it can be expressed as:

$$
\begin{aligned}
& W(A)=\int_{V_{1}+V_{2}} \frac{1}{2 \mu}\left[\left(\frac{\partial A}{\partial x}\right)^{2}+\left(\frac{\partial A}{\partial y}\right)^{2}\right] d x d y+\int_{V_{2}}\left(H_{c} \cos \theta \frac{\partial A}{\partial y}-H_{c} \sin \theta \frac{\partial A}{\partial x}\right) d x d y \\
& -\int_{V_{1}} J_{c} \bullet A d x d y+\int_{V_{2}} \frac{\mu}{2}\left(H_{c}\right)^{2} d \tau=\min
\end{aligned}
$$

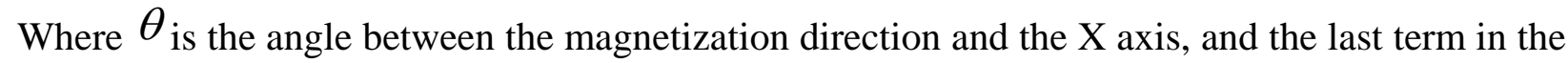
above equation is constant and does not work for the extremum.

When the mutative problem is discretized in the permanent magnet direct process, it can be found that the rest of the equation (4) is the same as the constant magnetic field in the absence of the permanent magnet except for the second term, so the discrete format is the same. Only the second part is newly added, and the corresponding discrete formula is derived as follows:

$$
W(A)=-\int_{V_{2}}\left(H_{c} \cos \theta \frac{\partial A}{\partial y}-H_{c} \sin \theta \frac{\partial A}{\partial x}\right) d x d y
$$

The first order partial derivative of the magnetic field for each node:

$$
\frac{\partial W_{e}^{\prime}}{\partial A_{k}}=-\frac{1}{2}\left(c_{k} H_{c} \cos \theta-b_{k} H_{c} \sin \theta\right) \quad k=i, j, m
$$


Where, $c_{k} 、 b_{k}$ is element discrete value coefficient of Two - dimensional plane field.

This is a constant that is independent of the magnetic field of the node and the following equation is superimposed on the right term of the node equation:

$$
P_{k}=\frac{1}{2}\left(c_{k} H_{c} \cos \theta-b_{k} H_{c} \sin \theta\right)
$$

This is the magnetic field with the node regardless of whether the uniform magnetization, as long as the permanent magnet magnetization around the calculation of coercivity known, can be calculated.

\section{Finite Element Analysis of Permanent Magnet DC Motor}

When the motor is unloaded, there is no current in the armature winding, only the magnetic field generated by the permanent magnet. Ignoring the motor's axial magnetic field changes, using the two-dimensional field analysis, the vector magnetic A has only the Z-axis component, which satisfies the following Poisson equation:

$$
\left\{\begin{array}{l}
\Omega: \frac{\partial}{\partial x}\left(v \frac{\partial A}{\partial x}\right)+\frac{\partial}{\partial y}\left(v \frac{\partial A}{\partial y}\right)=0 \\
S_{1}: A=0 \\
L: v_{1} \frac{\partial A}{\partial n}=v_{2} \frac{\partial A}{\partial n}-J_{m}
\end{array}\right.
$$

Due to the existence of the alveolar, so that the permanent magnet and the armature surface of the opposite side of the air gap is not uniform, resulting in reluctance torque, causing the motor output torque ripple, the motor winding is not energized when still exist, Called cogging torque. Cogging torque can cause vibration and noise. In some applications, the motor has a good smoothness and a certain limit on torque ripple. Therefore, it is important to study the cogging torque accurately and to study the measures to reduce the cogging torque. Cogging torque is calculated by making the armature current zero, only permanent magnet excitation, in different rotor position of the electromagnetic field finite element calculation, obtained cogging torque with the rotor position changes. Calculate the cogging torque to deal with rotor motion problems and the precise calculation of electromagnetic torque ${ }^{[8-9]}$.

$$
\left\{\begin{array}{l}
\Omega: \frac{\partial}{\partial x}\left(v \frac{\partial A}{\partial x}\right)+\frac{\partial}{\partial y}\left(v \frac{\partial A}{\partial y}\right)=-J_{c} \\
S_{1}: A=0 \\
L: v_{1} \frac{\partial A}{\partial n}=v_{2} \frac{\partial A}{\partial n}-J_{m}
\end{array}\right.
$$

In this work, dynamic finite element method is used to calculate the load magnetic field, taking into account the effect of motor cogging and armature winding commutation. With regard to the handling of the rotor movement, discussed in the previous section, the following discussion discusses the variation law of the armature in the commutation process. First, according to the number of motor slots and winding pitch for slot number distribution, and then in accordance with the actual brush and the width of the commutation for commutation calculation, get the distribution of electricity and changes in the law. For the whole winding, due to structural symmetry, the same slot on the upper and 
lower coil side is always the same time, this time the situation is relatively simple. Low-power motor often used in two odd slot motor, such as three slots, five slots, seven slots, etc., at this time for the short winding. The short windings are more complex than the distance winding, and there is a coil in the same slot that is commutative and the other is not in the commutation case. The following for low-power motor commonly used in the two odd number of slot analysis ${ }^{[10-11]}$.

\section{Simulation verification}

According to the above analysis method, a 5-slot permanent magnet DC motor and a 7-slot motor were calculated.

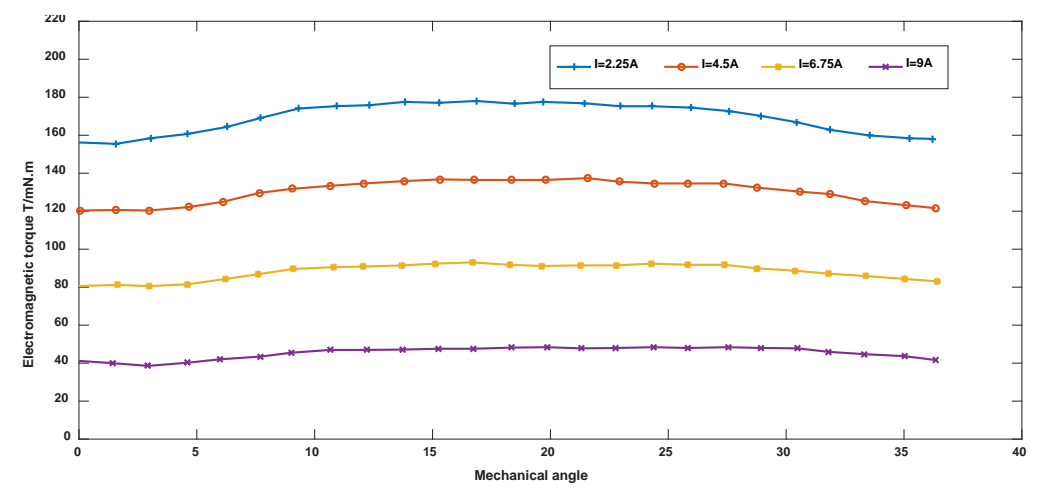

Fig.1. Electromagnetic Torque Curve of Five-slot Permanent Magnet DC Motor at Different Currents

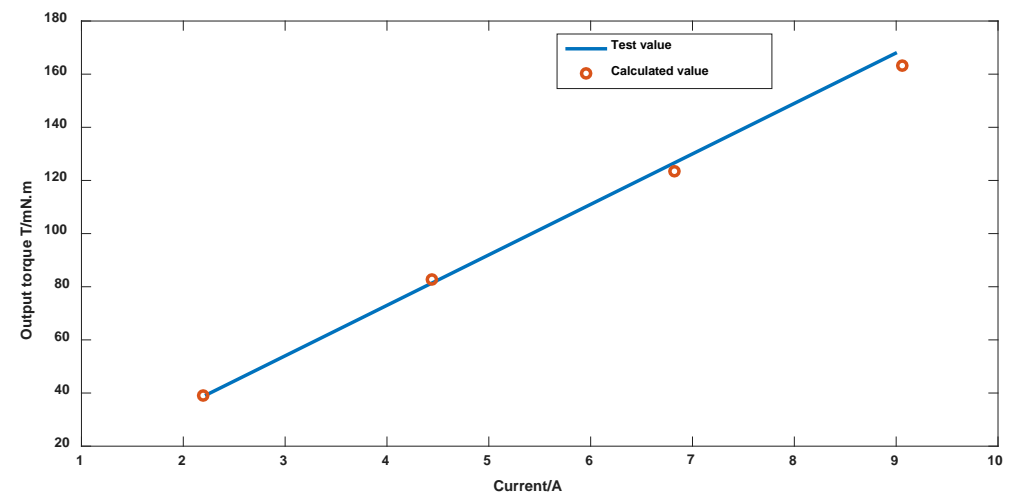

Fig.2. Output Torque Curve of Five - Slot Permanent Magnet DC Motor at Different Currents

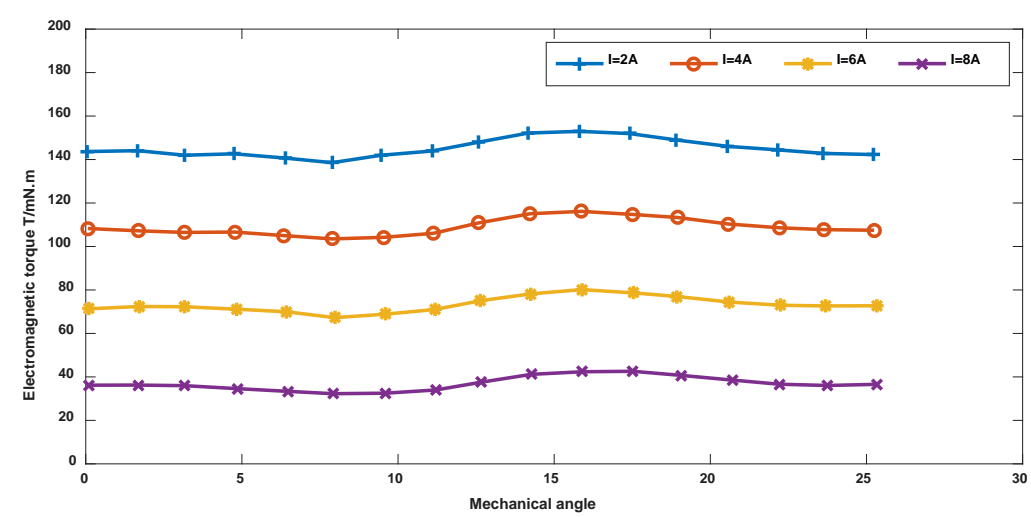

Fig.3. Electromagnetic Torque Curve of Seven-slot Permanent Magnet DC Motor at Different Currents 


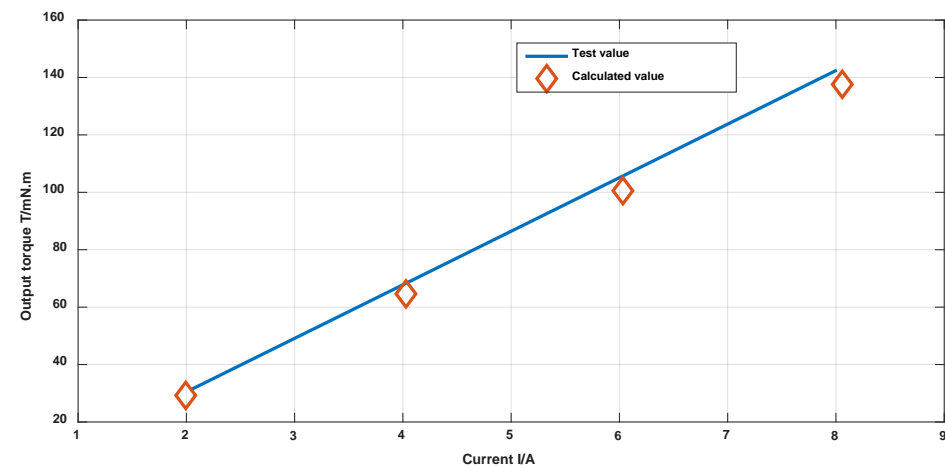

Fig.4. Output Torque Curve of Seven-Slot Permanent Magnet DC Motor at Different Currents

Fig. 1 and Fig. 3 shows the variation of the electromagnetic torque with the rotor position. Fig. 2 and Fig. 4 show the results of the calculation of the output torque with current And the calculated values and experimental values are given. The calculated value of the output torque is calculated by subtracting the idling torque from the electromagnetic torque, and the no-load torque is obtained from the test results. From the comparison results we can see that the finite element calculation results are very accurate.

\section{Summary}

Based on the number of motor slots and winding pitch, the slot number distribution is carried out, and then the commutation calculation is carried out according to the actual brush width, and the distribution and variation law are obtained. The interpolating motion boundary method is used to deal with the rotor motion, and the rotor is calculated according to the rotation speed of the rotor. The distribution of the electromagnetic distribution and the magnetic field distribution are calculated at each rotor position. The torque-torque characteristics obtained by the dynamic finite element analysis model are taken into account, taking into account the cogging effect and the torque ripple caused by the commutation, which is very important for the study of the performance of the permanent magnet DC motor. The analytical method used in this paper can be used to analyze the whole winding permanent magnet motor, can also analyze the ordinary DC motor.

\section{Acknowledgement}

This work was supported by Jiangxi Province Intellectual Property Soft Science Research Project (ZR201610).

\section{References}

[1] Zhu Z Q, Howe D, Bolte E, et al. Instantaneous magnetic field distribution in brushless permanent magnet DC motors. I. Open-circuit field[J]. IEEE transactions on magnetics, 1993, 29(1): 124-135.

[2] Zarko D, Ban D, Lipo T A. Analytical calculation of magnetic field distribution in the slotted air gap of a surface permanent-magnet motor using complex relative air-gap permeance[J]. IEEE Transactions on Magnetics, 2006, 42(7): 1828-1837.

[3] Zarko D, Ban D, Lipo T A. Analytical calculation of magnetic field distribution in the slotted air gap of a surface permanent-magnet motor using complex relative air-gap permeance[J]. IEEE Transactions on Magnetics, 2006, 42(7): 1828-1837.

[4] Zhu Z Q, Howe D, Chan C C. Improved analytical model for predicting the magnetic field distribution in brushless permanent-magnet machines[J]. IEEE Transactions on Magnetics, 2002, 38(1): 229-238.

[5] Rahman M A, Zhou P. Analysis of brushless permanent magnet synchronous motors[J]. IEEE Transactions on Industrial Electronics, 1996, 43(2): 256-267. 
[6] Kim U, Lieu D K. Magnetic field calculation in permanent magnet motors with rotor eccentricity: With slotting effect considered[J]. IEEE Transactions on Magnetics, 1998, 34(4): 2253-2266.

[7] Jabbar M A, Liu Z, Dong J. Time-stepping finite-element analysis for the dynamic performance of a permanent magnet synchronous motor[J]. IEEE transactions on magnetics, 2003, 39(5): 2621-2623.

[8] Zhu Z Q, Wu L J, Xia Z P. An accurate subdomain model for magnetic field computation in slotted surface-mounted permanent-magnet machines[J]. IEEE Transactions on Magnetics, 2010, 46(4): 1100-1115.

[9] Rahman M A, Zhou P. Determination of saturated parameters of PM motors using loading magnetic fields[J]. IEEE transactions on magnetics, 1991, 27(5): 3947-3950.

[10]Kim U, Lieu D K. Magnetic field calculation in permanent magnet motors with rotor eccentricity: Without slotting effect[J]. IEEE Transactions on Magnetics, 1998, 34(4): 2243-2252.

[11]Proca A B, Keyhani A, El-Antably A, et al. Analytical model for permanent magnet motors with surface mounted magnets[J]. IEEE transactions on energy conversion, 2003, 18(3): 386-391. 